\title{
PROBLEM TOPICALITY OF OFFSET ABSENCE ORDER INCREASE IN CONTROLLERS DURING CONTROL OF OBJECTS WITH VARYING TRANSMISSION COEFFICIENT
}

${ }^{1}$ Khobin V.A., ${ }^{2}$ Levinskyi M.V.

${ }^{1,2}$ Odessa National Academy of food technologies, Odessa

E-mail: ${ }^{2}$ MaxLevinskyi@gmail.com

${ }^{2}$ ORCID: $\underline{0000-0002-6544-5110}$

Copyright (C) 2014 by author and the journal "Automation technological and business - processes".

This work is licensed under the Creative Commons Attribution International License (CC BY).

http://creativecommons.org/licenses/by/4.0/

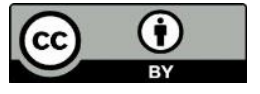

ONAFT

Open Access

DOI: $10.15673 /$

Abstract

Non-stationary control objects, especially with varying transmission coefficient, are fairly common in practice. Creation of automatic control systems (ACS) for them is topical issue. This task of creating ACS for these kind of control objects is more difficult than for stationary control objects (with constant parameters) because process value deviations relative to set point value are caused not only by coordinate disturbances but also by parametric ones. The issue of offset absence order increase impact on the levels of stabilization and reference tracking errors traditionally is dealt with when making synthesis of stationary ACS, particularly follow-up and program systems. For analytical solution of the non-stationary ACS analysis and synthesis problems, which contain control objects with varying transmission coefficient $k_{o}(t)$, it is necessary to utilize mathematical methods of linear systems with varying parameters. They are based on the concept of parametric transfer function. At the same time analytical calculation of stabilization and reference tracking errors, even for steady processes, is, apparently, extremely difficult, because there were found no examples of solution to this problem in technical publications. Computer modeling is considered more productive. It does not allow to obtain analytical expressions for control errors. But computer experiment goal planning allows to mark out their basic features and to evaluate effective methods of decreasing these mistakes.

Key words

Varying transmission coefficient, offset absence order increase, non-stationary control objects, automatic control system.

Introduction

Non-stationary control objects, especially with varying transmission coefficient, are fairly common in practice. Creation of ACS for them is topical issue. This task of creating ACS for these kind of objects is more difficult than for stationary control objects (with constant parameters) because process value $y(t)$ deviations relative to set point value $y^{s p}$ are caused not only by coordinate disturbances but also by parametric ones $k_{o}(t)$.

Block diagram of the simplest non-stationary ACS with varying transmission coefficient $k_{o}(t)$ of the control object is illustrated in fig. 1.

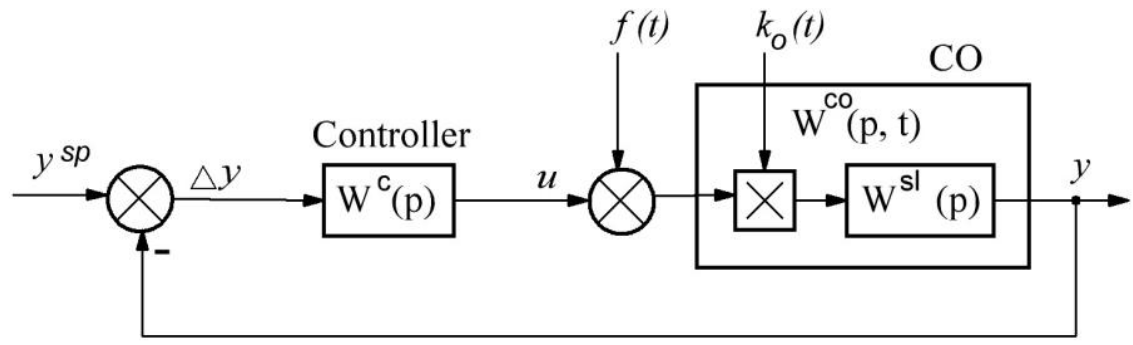

Fig. 1 Block diagram of ACS which includes control object with varying transmission coefficient 
Dynamic properties of this ACS are described by the set of linear differential equations:

$$
\left\{\begin{array}{l}
y(t)=k_{o}(t) \cdot W^{s l}(p) \cdot(u(t)+f(t))=k_{o}(t) \frac{R^{o}(p)}{Q^{o}(p)}(u(t)+f(t)) \\
u(t)=W^{c}(p) \cdot\left(y^{s p}-y(t)\right)
\end{array},\right.
$$

where $W^{s l}(p)$ - transfer function of the control object stationary linear part, $Q^{o}(p)$ - inherent operator, $R^{o}(p)$ - influence operator, $W^{c}(p)$ - transfer function of the controller, $p \equiv d / d t$ - differential operator.

The analysis of control errors $\Delta y(t)$ which occur in this system is given in the article. The problem topicality which is stated in the title is shown here.

Note that the ACS which is described by (1) is not adaptive. But this fact does not reduce research topicality of the additional control errors $\Delta y(t)$ which occur in the system due to $k_{o}(t)$ changes because they will develop not only in usual ACS but also in adaptive ones. Understanding the causes of these error occurrence will make it possible to find ways of reducing them. Particularly, as it follows from the title, utilizing offset absence order increase.

\section{Analysis of publications on the topic}

The issue of offset absence order increase impact on the levels of stabilization and reference tracking errors traditionally is dealt with when making synthesis of stationary ACS, particularly follow-up and program systems $[1,2]$. In this case the task of these errors calculation in steady processes is considered on the basis of the "Theorem about final value" of the Laplace transform.

For analytical solution of the non-stationary ACS analysis and synthesis problems, which contain control objects with varying transmission coefficient $k_{o}(t)$, it is necessary to utilize mathematical methods of linear systems with varying parameters [3]. They are based on the concept of parametric transfer function. At the same time analytical calculation of stabilization and reference tracking errors, even for steady processes, is, apparently, extremely difficult, because there were found no examples of this problem solution in technical publications. Approximate solution methods are proposed in [4] with the assumption that control object parameters changes take place relatively slow and it is possible to use method of "frozen" coefficients. For particular case, when control object transition coefficient changes according to linear dependence $k_{o}(t)=k_{o i n}+v_{k o} t$ and the rest parameters are stationary, expression for parametric transfer function has been obtained $[5,6]$ :

$$
W(s, t)=\frac{\left(k_{\text {oin }}+v_{k o} t\right) R^{o}(s)}{Q^{o}(s)}+v_{k o} R^{o}(s) \frac{d}{d t}\left(\frac{1}{Q^{o}(s)}\right)
$$

where s - complex variable of Laplace transform, $k_{\text {oin }}$-initial value of transition coefficient.

Further analysis and synthesis of the control system with such control object authors propose to carry out fixing the value of $t=$ const for particular value of the control object transmission coefficient using known methods for systems with constant parameters. In fact, it is offered to examine a set of partial solutions.

Computer modeling is considered more productive. It does not allow to obtain analytical expressions for control errors. But computer experiment goal planning allows to mark out their basic features and to evaluate effective methods of decreasing these mistakes.

The goal of this article - is to reveal the presence and features of additional stabilization and reference tracking errors which occur in ACS during control of control objects with varying transition coefficient and to evaluate the influence of ACS offset absence order on these errors.

Conditions for carrying out comparative research

ACS modeling was carried out according to block diagram in fig. 1 and for these model options:

- control object:

$$
W^{c o}(s, t)=k_{o}(t) \cdot W^{s l}(s)=k_{o}(t) \frac{1}{\left(T_{o} s+1\right)} \exp \left(-\tau_{o} s\right)
$$

where: $\tau_{o}=1, T_{o}=2-$ control object time delay and time constant values;

- parametric disturbance: 


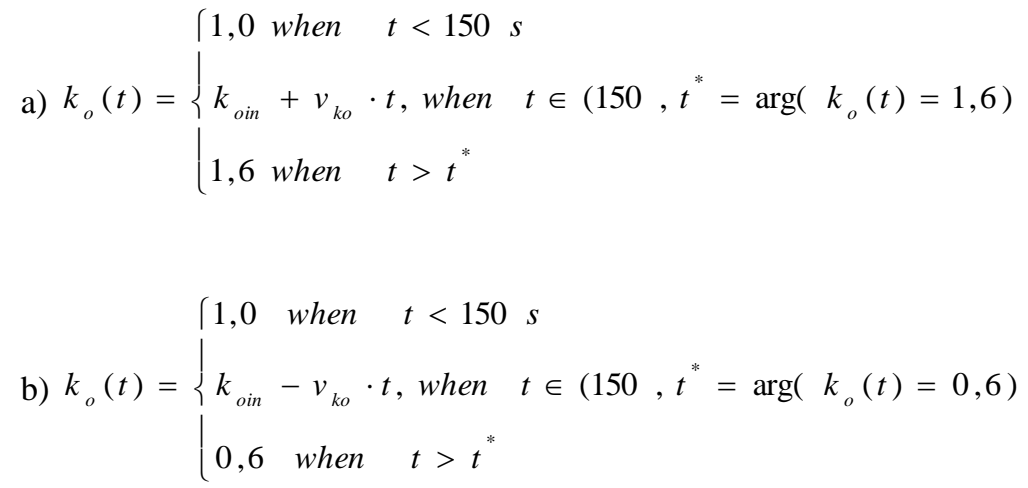

c) $k_{o}(t)=k_{\text {oin }}+0,3 \sin (\omega \cdot t)$,

where: $k_{\text {oin }}=1$ - transition coefficient initial value; $v_{k o}-k_{o}$ speed change; $\omega$ - frequency of harmonic oscillation;

- controller:

a) PI $-W^{c}(s)=k_{p}\left(1+1 / T_{i 1} s\right)$,

b) $\left.\mathrm{PII}^{2}-W^{c}(s)=k_{p}\left(1+1 / T_{i} s\right)+1 / T_{i 1} T_{i 2} s^{2}\right)$,

where $k_{p}=2,34-$ transition coefficient values; $T_{i l}=T_{i 2}=2,70$ - integrating constants values - parameters of controller which ensure acceptable transitional processes within all range of $k_{o}(t)$ change;

- coordinate disturbances in channel "f-y" $f(t)=m_{f}+\tilde{f}(t)$ :

a) $f(t)=0$,

b) $m_{f}=0, \quad S_{\tilde{f}}=1,762 /\left(\omega^{4}+0,0625\right)$,

- coordinate disturbances in channel " $\mathrm{y}^{\mathrm{sp}}-\mathrm{y} " y^{s p}(t)=m_{y^{s p}}+\tilde{y}^{s p}(t)$ :

a) $y^{s p}(t)=$ const

b) $m_{y^{s p}}=$ const $, \quad S_{\tilde{y}^{s p}}=0,028 /(\omega 4+0,0001)$

where: $m_{f}, m_{y^{s p}}$ - deterministic components of $f$ and $y^{s p}$ (mathematical expectation); $\tilde{f}(t), \tilde{y}^{s p}(t)$ - centered random components of $f$ and $y^{s p} ; S_{\tilde{f}}, S_{\tilde{y}^{s p}}$ - the spectral densities of random components.

Specific conditions of carrying out computer experiments and their results are reflected in the figures field and in the summary table 1 .

The results of comparative research

Non-stationary ACS free motion research during linear changing of parametric disturbance for models: (3), (4), (5), (7), (8), (9), (11). Modeling fragments are shown in fig. 2, 3. 


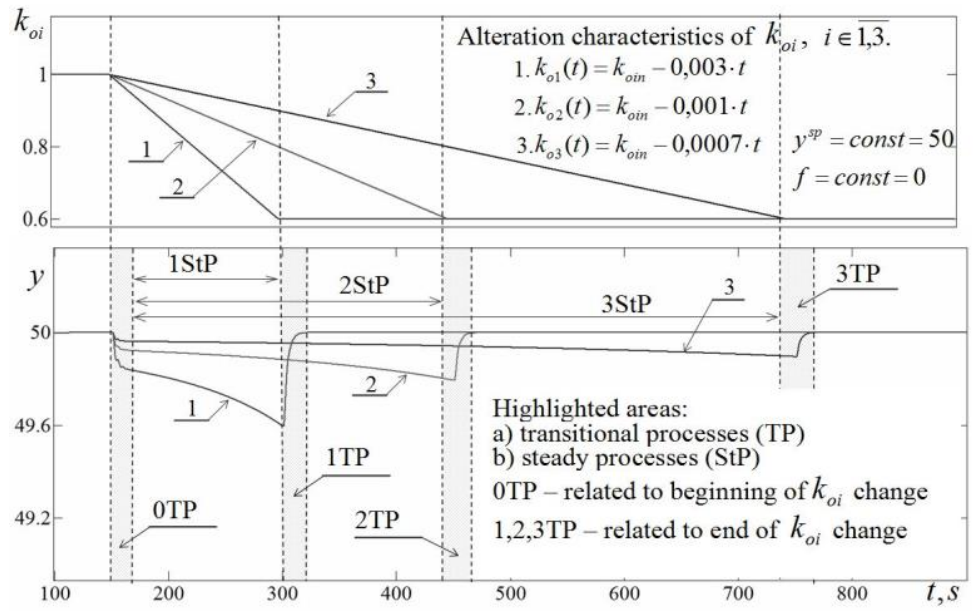

a)

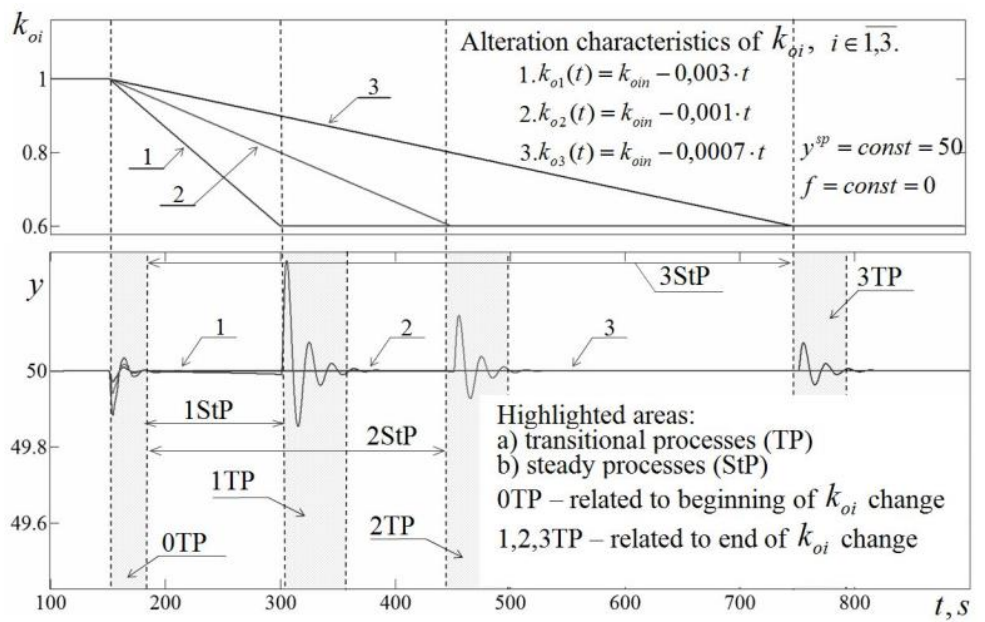

b)

Fig. 2 - ACS controlled variable response with PI- (a) and PII ${ }^{2}$-controller (b) during linear decrease of control object transition

$$
\text { coefficient } k_{o} \text { and with fixed coordinate disturbances } y^{s p} \text { and } f
$$

In ACS free motion with linear changing parametric disturbances, as follows from fig. 2, in $y(t)$ changes we can separate transient processes and steady processes.

Occurrence of transient processes can be explained with the fact that $k_{o}(t)$ changes take place in finite time interval (see (4), (5)), at the beginning and at the end of which speed $v_{k o}$ of $k_{o}(t)$ changes equals to zero. Transient processes areas are marked with grey color. In system with the first order of offset absence transient processes are aperiodic, with the second order - oscillating and controlled variable maximum dynamic deviation $y(t)$ is in proportion to transition coefficient $k_{o}(t)$ changing speed $v_{k o}$.

During linear changing of $k_{o}(t)$ processes in ACS have steady behavior. In this mode in system with first order of offset absence steady errors $\Delta y(t)$ of controlled variable occur. The value of these errors is in proportion to $k_{o}(t)$ speed change $v_{k o}$. And $\Delta y(t)$ changes are nonlinear and progress with $k o(t)$ change. In system with the second order of offset absence, as follows from fig. $2 b$, steady errors are close to zero. 

www.journal-atbp.com

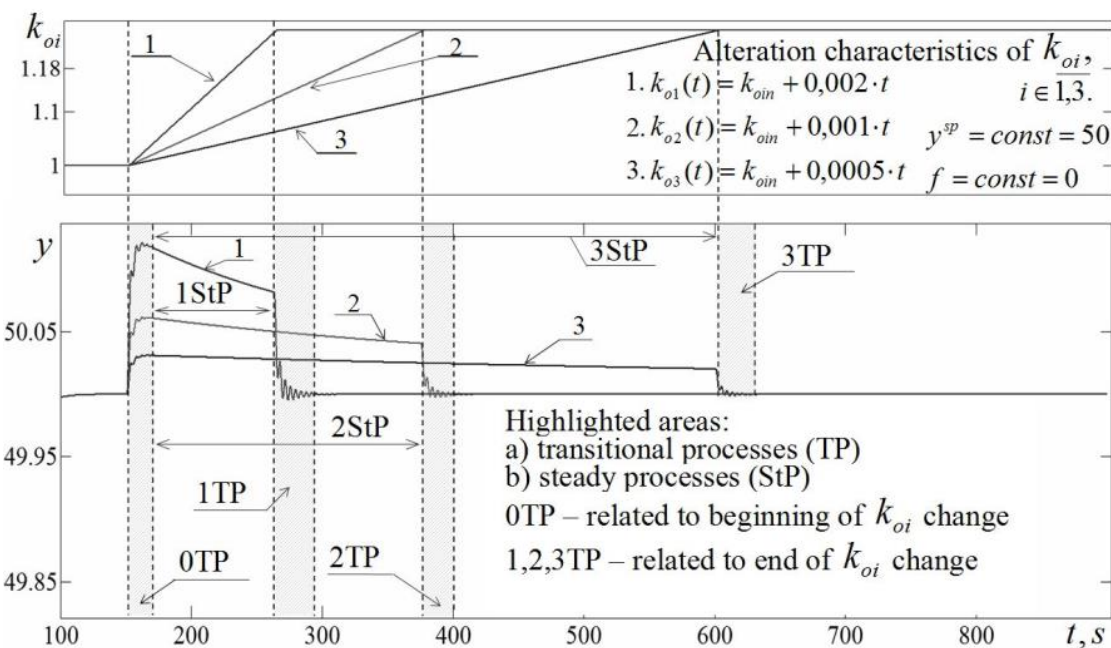

a)

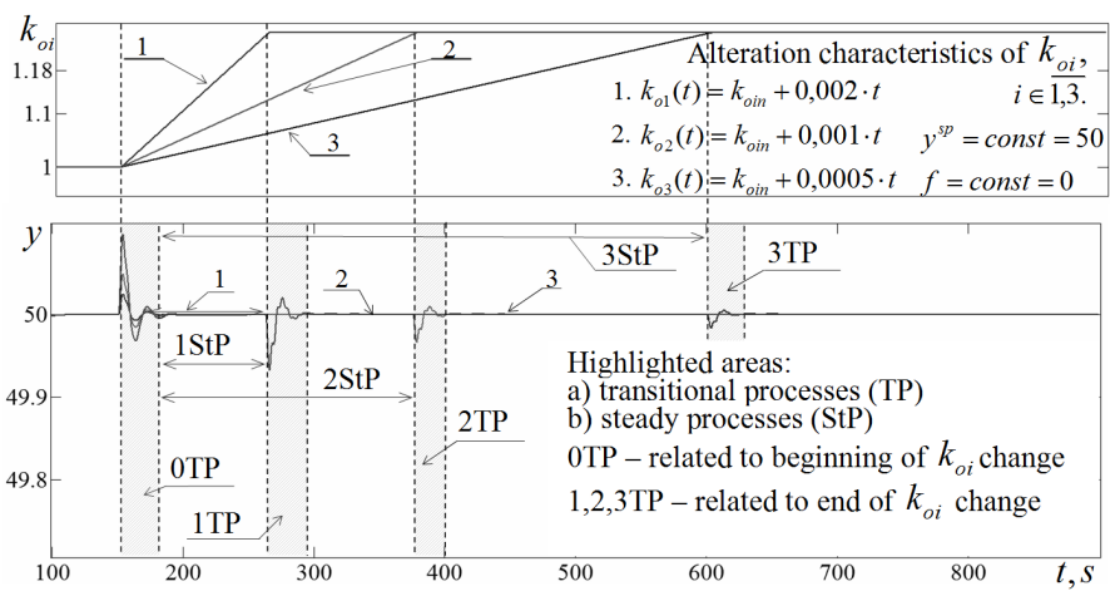

b)

Fig. 3 - ACS controlled variable response with PI- (a) and PII ${ }^{2}$-controller (b) during linear increase of control object transition coefficient $k_{o}$ and with fixed coordinate disturbances $y^{\text {sp }}$ and $f$

Similar ACS research has been done during linear increase of control object transition coefficient $k_{o}(t)$. When comparing fig. $2 \mathrm{a}$ and $3 \mathrm{a}$, we can notice, that with linear $k_{o}(t)$ change in ACS with first order of offset absence steady error $\Delta y(t)$ of controlled variable have different behavior: in the first case it increases and in the second case - decreases.

Non-stationary ACS free motion research with harmonic changing of parametric disturbance has been done for models: (3), (6), (7), (8), (9), (11). Modeling fragments are shown in fig. 4. 


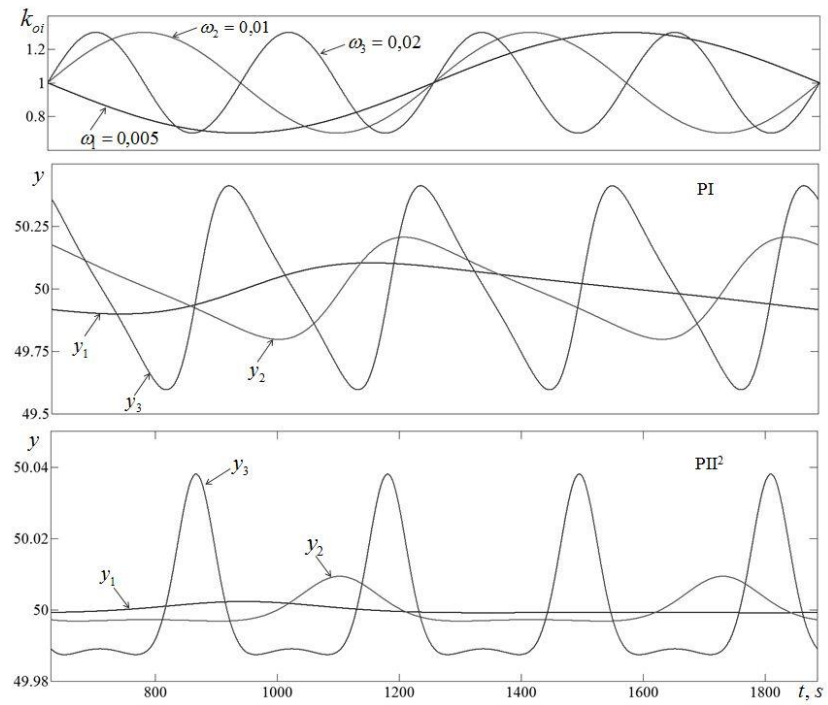

Fig. 4 - ACS controlled variable response with PI and PII $^{2}$-controller with harmonically changing control object transition coefficient $k_{o i}=k_{o i n}+0,3 \cdot \sin \left(\omega_{i} t\right), i \in 1,3$ and with fixed coordinate disturbances $y^{s p}$ and $f$

Transient processes which occur at the beginning and at the end of parametric disturbance $k_{o}(t)$ change are not included in the time interval which is shown in fig. 4. In this case ACS operates at steady state. In system with first order of offset absence errors $\Delta y(t)$ occur and their magnitude is in proportion to oscillation frequency $\omega$ of $k_{o}(t)$. Also unsymmetry of controlled variable $y(t)$ oscillations can be seen because during transition coefficient $k_{o}(t)$ increase errors $\Delta y(t)$ are suppressed more effectively and during decrease - on the contrary.

ACS offset absence order increase from first to second leads to considerable errors $\Delta y(t)$ suppress and they occur only at the moments of $y(t)$ derivative sign change during decreasing of $k o(t)$.

Non-stationary ACS forced motion research with parametric disturbance harmonic change and random change of coordinate disturbances has been done for models: (3), (6), (7), (8), (10), (11). Modeling fragments are shown in fig. 5.
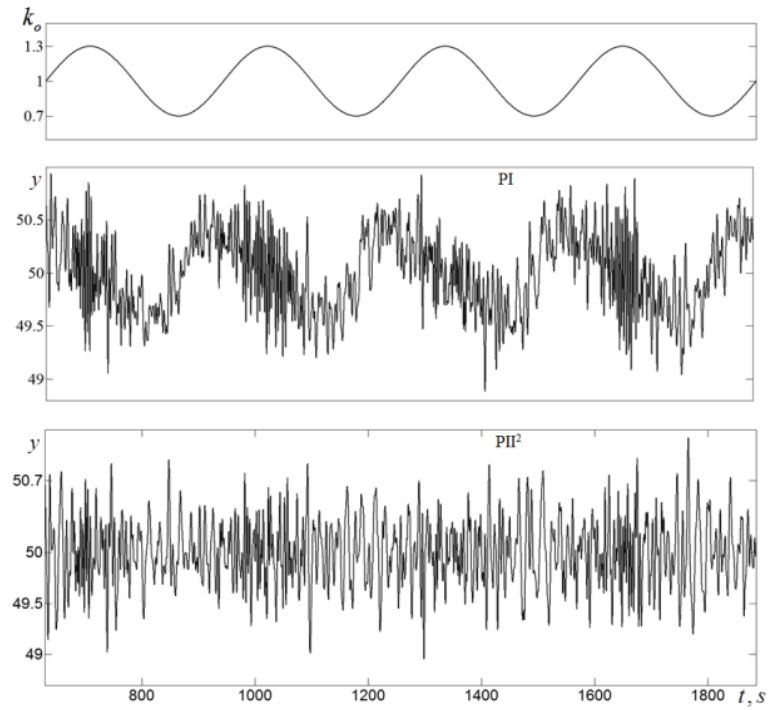

Fig. 5 - ACS controlled variable response with PI and PII $^{2}$-controller with harmonically changing control object transition coefficient $k_{o}=k_{o i n}+0,3 \cdot \sin (0,02 \cdot t)$ and with fixed disturbance $y^{s p}$ and with standing random disturbances $f(t)$

This example is closer to actual conditions of ACS operation when coordinate disturbances of random nature $f(t)$ make impact on control object. In ACS with the first order of offset absence parametric disturbances $k_{o}(t)$ lead to occurrence of additional low-frequency components in control variable $y(t)$ changes.

In ACS with the second order of offset absence the average value of stochastic process $y(t)$ is less deviant from set point value. 
Generalized experiment results for the case of harmonic $k_{o}(t)$ change are shown in the table 1. ACS operating accuracy with the first(1) and the second(2) order of offset absence in steady state is convenient to compare using proportion of their errors $\overline{\Delta y}^{(2)} \overline{\Delta y_{i}}$

errors $\Delta y_{i} / \Delta y_{i}$, which can be determined from the expression:

$$
\overline{\Delta y}_{i}=\frac{\sqrt{\int_{t_{b}}^{t_{e}} \Delta y_{i}^{2}(t) d t}}{2 \pi / \omega_{i}}, \text { where } t_{b}=2 \pi / \omega_{i}, t_{e}=4 \pi / \omega_{i}, i \in \overline{1,3} .
$$

Table 1. Comparative research results of ACS operating quality with the first and the second order of offset absence

\begin{tabular}{|c|c|c|c|c|c|c|c|c|c|}
\hline \multirow{3}{*}{ 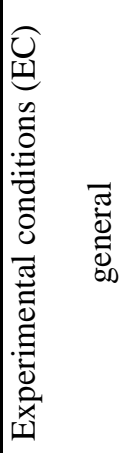 } & \multicolumn{9}{|c|}{ Parametric disturbance $k_{o i}(t)=k_{o i n}+0,3 \cdot \sin \left(\omega_{i} t\right), i \in \overline{1,3}$. } \\
\hline & \multicolumn{3}{|c|}{$\omega_{1}=0,005 \frac{\mathrm{rad}}{\mathrm{s}}$} & \multicolumn{3}{|c|}{$\omega_{2}=0,01 \frac{\mathrm{rad}}{\mathrm{s}}$} & \multicolumn{3}{|c|}{$\omega_{3}=0,02 \frac{\mathrm{rad}}{\mathrm{s}}$} \\
\hline & \multicolumn{2}{|c|}{$\overline{\Delta y}_{i}^{(2)}, \overline{\Delta y}_{i}^{(1)}$} & \multicolumn{7}{|c|}{ - error evaluation in ACS with the first and the second order of offset absence } \\
\hline $\bar{U}$ & \multicolumn{9}{|c|}{$\begin{array}{l}\text { 1. External disturbance } f(t)=0 \text {, } \\
\text { set point value } y^{s p}=\text { const }\end{array}$} \\
\hline$y^{s p}$ & $\overline{\Delta y}_{1}^{(1)}$ & $\overline{\Delta y}_{1}^{(2)}$ & $\frac{\overline{\Delta y}_{1}^{(2)}}{\overline{\Delta y_{1}}}$ & $\overline{\Delta y}_{1}^{(1)}$ & $\overline{\Delta y}_{1}^{(2)}$ & $\frac{\overline{\Delta y}_{1}^{(2)}}{\overline{\Delta y_{1}}}$ & $\overline{\Delta y}_{1}^{(1)}$ & $\overline{\Delta y}_{1}^{(2)}$ & $\frac{\overline{\Delta y}_{1}^{(2)}}{\overline{\Delta y_{1}}}{ }^{(1)}$ \\
\hline 20 & 0,0008 & 0,000012 & 0,015 & 0,0015 & 0,000047 & 0,03 & 0,0031 & 0,0002 & 0,061 \\
\hline 30 & 0,0011 & 0,000018 & 0,016 & 0,0023 & 0,000071 & 0,03 & 0,0046 & 0,0003 & 0,061 \\
\hline 40 & 0,0015 & 0,000024 & 0,016 & 0,0031 & 0,000094 & 0,03 & 0,0061 & 0,0004 & 0,062 \\
\hline 50 & 0,0019 & 0,000029 & 0,015 & 0,0038 & 0,000120 & 0,03 & 0,0076 & 0,0005 & 0,062 \\
\hline 60 & 0,0023 & 0,000035 & 0,015 & 0,0046 & 0,000140 & 0,03 & 0,0092 & 0,0006 & 0,062 \\
\hline$\bigcup_{\text {UI }}^{N}$ & \multicolumn{9}{|c|}{ 2. External disturbance $f(t)=m_{f}+\tilde{f}(t), m_{f}=0, S_{\tilde{f}}=1,762 /\left(\omega^{4}+0,0625\right)$} \\
\hline$y^{s p}$ & $\overline{\Delta y}_{2}^{(1)}$ & $\overline{\Delta y}_{2}^{(2)}$ & $\frac{\overline{\Delta y}_{2}^{(2)}}{\overline{\Delta y_{2}}}$ & $\overline{\Delta y_{2}}$ & $\overline{\Delta y}_{2}^{(2)}$ & $\frac{\overline{\Delta y}_{2}^{(2)}}{\overline{\Delta y_{2}}}$ & $\overline{\Delta y}_{2}^{(1)}$ & $\overline{\Delta y}_{2}^{(2)}$ & $\frac{\overline{\Delta y}_{2}{ }^{(2)}}{\overline{\Delta y}_{2}{ }^{(1)}}$ \\
\hline 20 & 0,0077 & 0,0092 & 1,19 & 0,0071 & 0,0092 & 1,30 & 0,0077 & 0,0092 & 1,19 \\
\hline 30 & 0,0077 & 0,0092 & 1,19 & 0,0073 & 0,0092 & 1,26 & 0,0084 & 0,0092 & 1,10 \\
\hline
\end{tabular}




\begin{tabular}{|c|c|c|c|c|c|c|c|c|c|}
\hline 40 & 0,0078 & 0,0092 & 1,18 & 0,0076 & 0,0092 & 1,21 & 0,0093 & 0,0092 & 0,99 \\
\hline 50 & 0,0079 & 0,0092 & 1,16 & 0,0079 & 0,0092 & 1,16 & 0,0100 & 0,0092 & 0,92 \\
\hline 60 & 0,0080 & 0,0092 & 1,15 & 0,0083 & 0,0092 & 1,11 & 0,0120 & 0,0092 & 0,77 \\
\hline$\bigcup_{i}^{m}$ & \multicolumn{9}{|c|}{$\begin{array}{l}\text { 3. External disturbance } f(t)=0 \text {, set point value } y^{s p}(t)=m_{y^{\text {sp }}}+\tilde{y}^{s p}(t) \text {, } \\
\qquad m_{y^{\text {sp }}}=\text { const }, \quad S_{\tilde{y}^{\text {mp }}}=0,028 /\left(\omega^{4}+0,0001\right)\end{array}$} \\
\hline$m_{y^{s p}}$ & $\overline{\Delta y}_{3}^{(1)}$ & $\overline{\Delta y}_{3}{ }^{(2)}$ & $\frac{\overline{\Delta y}_{3}{ }^{(2)}}{\overline{\Delta y}_{3}^{(1)}}$ & $\overline{\Delta y}_{3}^{(1)}$ & $\overline{\Delta y}_{3}^{(2)}$ & $\frac{\overline{\Delta y}_{3}^{(2)}}{\overline{\Delta y}_{3}^{(1)}}$ & $\overline{\Delta y}_{3}^{(1)}$ & $\overline{\Delta y}_{3}^{(2)}$ & $\frac{\overline{\Delta y}_{3}^{(2)}}{\overline{\Delta y_{3}}}$ \\
\hline 20 & 0,0048 & 0,0021 & 0,44 & 0,0049 & 0,0022 & 0,45 & 0,0056 & 0,0023 & 0,41 \\
\hline 30 & 0,0049 & 0,0021 & 0,43 & 0,0052 & 0,0022 & 0,42 & 0,0066 & 0,0023 & 0,35 \\
\hline 40 & 0,0050 & 0,0021 & 0,42 & 0,0056 & 0,0022 & 0,39 & 0,0077 & 0,0023 & 0,30 \\
\hline 50 & 0,0051 & 0,0021 & 0,41 & 0,0060 & 0,0022 & 0,37 & 0,0089 & 0,0023 & 0,26 \\
\hline 60 & 0,0053 & 0,0021 & 0,40 & 0,0066 & 0,0022 & 0,33 & 0,0100 & 0,0024 & 0,24 \\
\hline
\end{tabular}

The analysis of the table indicate that the ACS errors values with the first order of offset absence when controlling of the control object with varying transition coefficient is in proportion to set point value and, therefore, is in proportion to according control variable average value and to control object transition coefficient speed change. In most cases, as experiments show, ACS with the second order of offset absence show better operating quality.

It should be noted that all experiments were carried out in the specific range of control object transition coefficient change where ACS was stable. For control objects in which $k_{o}(t)$ changes in wider range, controller transition coefficient selftuning loop should be added to the ACS structure. For such systems the task of ACS controller offset absence order increase remains relevant.

\section{Conclusions}

1. In ACS with the first order of offset absence during control of object with linear increase of transition coefficient, additional control errors occur, which in their nature are similar to speed errors of stationary ACS which occur as a consequence of coordinate disturbances linear change. The values of these additional errors are in proportion to control variable average value and to control object transition coefficient change speed.

2. It is possible to compensate the errors which occur as a consequence of parametric disturbances impact with increase of offset absence order, e.g. by addition of one more integral component to the controller. Reasonability decision of such increase should be made taking into consideration concrete conditions of ACS operation.

\section{Referenses}

[1] Topcheev U.I. Atlas dlya proektirovaniya sistem avtomaticheskogo regulirovaniya/ U.I. Topcheev. - M.: Mashinostroenie, 1989. - 752s

[2] Besekerskiy V.A. Teoriya sistem avtomaticheskogo regulirovaniya/ V.A. Besekerskiy, E.P. Popov. - M.: Nauka, 1972. $768 \mathrm{~s}$.

[3] Solodovnikov V.V. Technicheskaya kibernetika. Teoriya avtomaticheskogo regulirovaniya/ V.V. Solodovnikov. - M.: Mashinostroenie, 1969. - 607s.

[4] Barkovskiy V.V. Metodi sinteza sistem upravleniya/ V.V. Barkovskyi, V.N. Zaharov, A.S. Shatalov. - M.: Mashinostroenie, 1969. - $323 \mathrm{~s}$.

[5] Rudnizkiy B.E. Opredelenie peredatochnih funkziy nekotorih systems peremennimi parametrami/ B.E. Rudnizkiy. - M.: Avtomatika i telemehanika, 1960. - $1565-1575 \mathrm{~s}$.

[6] Semenihin V.K. Peredatochnie funkzii sistem avtomaticheskogo regulirovaniya s lineino izmenyaushimisya parametrami. M.: Avtomatika i telemechanika, 1962. - 1421-1430 s. 